\title{
New heritable ATRX mutation identified by whole exome sequencing and review
}

\author{
Zahra Shahbazi ${ }^{1,2}$, Golaleh Rostami ${ }^{1}$ and Mohammad Hamid ${ }^{1 *}$ (i)
}

\begin{abstract}
Background: The mutations in the ATRX gene have been shown to cause two types of disorders: inherited mutations lead to alpha thalassemia X-linked mental retardation (ATR-X) syndrome and acquired somatic mutations cause alpha thalassemia myelodysplastic syndrome (ATMDS). Here we report a case of ATRX gene mutation without completely features of ATR-X or ATMDS syndromes. Moreover we review previous reports of ATRX gene mutations in both ATR-X syndrome and ATMDS.

Methods: After sample collection and DNA extraction, whole exome sequencing was performed using Illumina HiSeq PE150 apparatus. The results were confirmed using Sanger sequencing for the patients and his relatives. Literature review was performed based on the published data in Web of science, Science direct, Springer link and Pubmed databases.

Results: We identified a hemizygous missense ATRX gene mutation (ATRX, c.2388A > C, p. K796N) as a new diseasecausing variant in the patient, heterozygous situation for his mother and his father was hemizygous for wild type allele. The literatures of patients were reviewed regarding the ATR-X syndrome.

Conclusions: According to previous findings, inherited ATRX mutations are associated with a broad spectrum of clinical presentations. Therefore a person with a mild a-thalassemia phenotype may also has mutation in ATRX gene. Accordingly, it is critical for geneticist and physicians to increase awareness in molecular diagnosis of a-thalassemia patients.
\end{abstract}

Keywords: Alpha-thalassemia, ATR-X syndrome, Myelodysplastic syndrome, Whole-exome sequencing

\section{Background}

Myelodysplastic syndrome (MDS) is a heterogeneous group of clonal hematopoietic stem cell disorders characterized by peripheral blood cytopenias and microcytic red blood cell (RBC) indices in the absence of iron deficiency as a result of ineffectual erythropoiesis $[1,2]$. In contrast to the generic macrocytic anemia in MDS [3], the anemia of ATMDS ( $\alpha$-thalassemia myelodysplastic syndrome; OMIM catalog \#300448) is often specify as microcytic and hypochromic [2]. About $25 \%$ of patients with alpha thalassemia myelodysplastic syndrome

\footnotetext{
*Correspondence: hamid143@yahoo.com

1 Department of Molecular Medicine, Biotechnology Research Center,

Pasteur Institute of Iran, Tehran, Iran

Full list of author information is available at the end of the article
}

(ATMDS) will progress to acute myelogenous leukemia (AML), the risk of this progression appears to be similar to that of the MDS patient population [4]. A high ratio of MDS patients have acquired somatic mutations in the chromatin remodeling factor ATRX [2]. The ATRX gene encodes a member of the SWI2/SNF2 (Switching defective/Sucrose nonfermenting) family of proteins [5] that may act as a transcriptional factor and plays a significant role in the epigenetic regulation of gene expression [6]. The unusual $\alpha$-thalassemia and microcytosis in the ground of MDS is due to a decreased synthesis of $\alpha$-globin related to a dramatic down regulation of $\alpha$-globin gene expression by inactivating mutation in the ATRX gene [7]. On the other hand, inherited mutations in the ATRX gene, have been shown to cause an alpha thalassemia X-linked mental retardation (ATR-X) 
syndrome, ( $\alpha$ thalassemia with retardation encoded on the X chromosome; OMIM catalog \#301040). In this disease mild $\alpha$ - thalassemia have been found in boys with severe mental retardation, facial dysmorphism, and urogenital abnormalities $[8,9]$. Here we report a new hereditary missense mutation in exon 9 of the ATRX gene in a young MDS patient with mild $\alpha$-thalassemia. We also reviewed reports of ATRX gene mutations in databases and previous accessible publications.

\section{Materials and methods}

\section{Sample collection and DNA extraction}

Relevant informed consent was obtained from the patient. Two milliliters of peripheral blood were collected and DNA was extracted from patient's peripheral blood leukocytes by salting out method. High quality DNA samples, with the OD260/280 ratio of $1.8-2.0$, at the concentration of $100 \mathrm{ng} / \mu \mathrm{l}$ were used for further analysis. This study was also reviewed and approved by the Ethics Committee of Pasteur Institute of Iran.

Table 1 PCR primers used for the PCR-sequencing of exon 9 of ATRX gene

\begin{tabular}{ll}
\hline Primer name & Primer sequence $\mathbf{5}^{\prime} \mathbf{>} \mathbf{3}^{\prime}$ \\
\hline ATRX-F & GATGAGTCACAGTTCTTCTTCAG \\
ATRX-R & TCTTTTTTTGGTGGTTCTGGC \\
\hline
\end{tabular}

\section{Molecular investigation}

In order to investigate causing gene, exome sequencing was performed using Illumina HiSeq PE150 (Novagen company, Beijing, China) by SureSelect Human All Exon V6 Kit (Agilent) with an average coverage of $100 x$-fold and 150 bp read-length.

In order to approve founded variation, we performed Polymerase chain reaction (PCR) and Sanger sequencing method on the patient's DNA sample and his relatives (The primers sequences are available in Table 1). PCR amplification was carried out in a final volume of $30 \mu \mathrm{l} \mathrm{PCR} \mathrm{master} \mathrm{mix} \mathrm{(Ampliqon)} \mathrm{containing} \mathrm{100-}$ $200 \mathrm{ng}$ genomic DNA, and $10 \mathrm{pmol}$ of each primer.

Evaluation of PCR products was done by electrophoresis on $1 \%$ agarose gel. Finally, the PCR products were sequenced at Macrogene company (South Korea). The sequencing results were analyzed by Chromas (V2.6) and CLC workbench (V5.5 CLC bio) software and NM_000489.3 was used as reference sequence for ATRX (Fig. 1). The pathogenicity of the variant was reevaluated using the updated guideline for interpretation of molecular sequencing by the American College of Medical Genetics and Genomics (ACMG) considering the allele frequency in the population database, immunological/functional data, familial segregation and parental genotype (https://www.acmg.net/).



Fig. 1 ATRX gene Sanger sequencing result for the proband shows hemizygous genotype for mutant $C$ allele (A), patient's father is normal (B) and his mother is heterozygous (C) 


\section{Data collection}

Information on previous reports of pathogenic variants in the $A T R X$ gene was obtained by examining databases such as Web of science (www.webofknowledge.com), Science direct (www.sciencedirect.com), Springer link (https://link.springer.com) and Pubmed (www.ncbi.nlm. nih.gov/pubmed). We also used bioinformatics databases like NCBI (www.ncbi.nlm.nih.gov), Ensembl (www. ensembl.org), HGMD (www.hgmd.cf.ac.uk), COSMIC (https://cancer.sanger.ac.uk/cosmic), UniProt (www. uniprot.org) and OMIM (https://www.omim.org/) in writing this review article.

\section{Results}

\section{Case report}

Family originates from Baghdad the capital of Iraq and is of Arab ethnicity with consanguineous parents. A proband 29-year-old male with $167 \mathrm{~cm}$ height referred because of gastrointestinal signs, was found to have normocytic anemia (low hemoglobin (Hgb) $(5.6 \mathrm{~g} / \mathrm{dl}$ ), low mean corpuscular hemoglobin $(\mathrm{MCH})(26.8 \mathrm{pg})$ and haematocrit percent (18.3\%). He was normal for some quantities including mean corpuscular volume (MCV) (96.4fL), white blood cell (WBC) count $\left(4.3 \times 10^{3} / \mathrm{mm}^{3}\right)$, platelet count (PLT) $\left(199 \times 10^{3} / \mathrm{mm}^{3)}\right.$ and lymphocyte, monocyte, eosinophil, basophil percent $(36.9 \%$, 4.5\%, $0.8 \%, 0.2$ respectively). Interestingly his $\mathrm{RBC}$ count was low $2.34 \times 10^{6} / \mathrm{mm}^{3}$, as one of the hallmarks of MDS. He had no family history of thalassemia and no evidence of iron deficiency or other hemoglobinopathy. On physical examination, there was no sign of mental retardation, facial dysmorphism, urogenital abnormalities and palpable lymphadenopathy or splenomegaly. Pelvic ultrasound results showed normal size and texture of kidneys, no dilatation of PCS, no evidence of stones, normal thickness of the cortex, normal thickness of the wall of bladder, no evidence of stone in bladder, normal prostate, no abdominal or pelvic free fluid or mass.

Whole Exome Sequencing analysis identified A>C transition at c.2388 position in codon 796 of exon 9 of the $A T R X$ gene leading to the putative missense p.K796N mutation. To the best of our knowledge, the mutation has never been described before in MDS in the Single Nucleotide Polymorphism Database (https://www.ncbi. nlm.nih.gov), Ensemble (http://m.ensemble.org) and HGMD (http://www.hgmd.cf.ac.uk). This variation is likely pathogenic variant based on ACMG. The mutation is predicted to be damaging by PredictSNP, Sorting Intolerant from Tolerant (SIFT) and Polymorphism Phenotyping-2 (PolyPhen-2) softwares (Table 2). Verification Sanger sequencing results showed hemizygous alteration in patient, heterozygous situation for his mother (a wild
Table 2 Interpretation scores for the pathogenicity of p.K796N variant in ATRX gene

\begin{tabular}{lllll}
\hline Software & PredictSNP & Polyphen-1 & Polyphen-2 & SIFT \\
\hline Score & $51 \%$ & $59 \%$ & $43 \%$ & $53 \%$ \\
& Deleterious & Deleterious & Deleterious & Deleterious \\
\hline
\end{tabular}

type allele and an allele with a single mutation p.K796N) and his father was hemizygous for wild type allele (Fig. 1).

Literature review for ATR-X syndrome showed Nonhomogeneous distribution of mutations in different domains of ATRX gene. According to the review of this study, most of the previously reported mutations which lead to clinical consequences of ATR-X syndrome were occur in conserved domain of the ATRX gene including ADD, ATPase and C-terminal domains (Fig. 2). Moreover there was more variety of mutation types reported in ATRX gene for ATR-X syndrome (Fig. 3). About ATMDS, there was a homogeneous distribution of mutations in different domains of $A T R X$ gene. In other words, there was no significant difference in the mutation distribution in different domains of $A T R X$ gene in ATMDS reported cases. Moreover there were multiple reports of mutations in spacer region between EZH2 and DAXX domains in some ATMDS patients, the region that we found our patient's mutation, while in the ATR-X syndrome no mutation was reported in this region. Also there was less variety of mutation types reported in $A T R X$ gene for ATMDS (Fig. 3).

Anyway in both diseases in the terms of variant types, missense variant was the most common, afterwards splicing, small deletion and nonsense variants were commonest types respectively (Fig. 3). According to the review of this study, most of mutations which lead to clinical consequences occurred in these conserved domains.

\section{Discussion}

MDS is a heterogeneous group of clonal hematopoietic stem cell disorders characterized by peripheral blood microcytic and cytopenias RBC indices as a result of ineffectual erythropoiesis in the absence of iron deficiency $[1$, 2]. Here we report a case of MDS without usual presentation of ATR-X syndrome or ATMDS. The patient was a male case with $A T R X$ hereditary mutation manifested mild $\alpha$-thalassemia and no sign of mental retardation, facial dysmorphism, and urogenital abnormalities as the signs of ATR-X syndrome. His mother was carrier and has a normal appearance and intellect, and no signs of $\alpha$-thalassemia. Our patient has shown neither the severe hematologic signs expected in ATMDS nor the widespread manifestations of the ATR-X syndrome, this may be due to the fact that the mutation in this patient occurs 


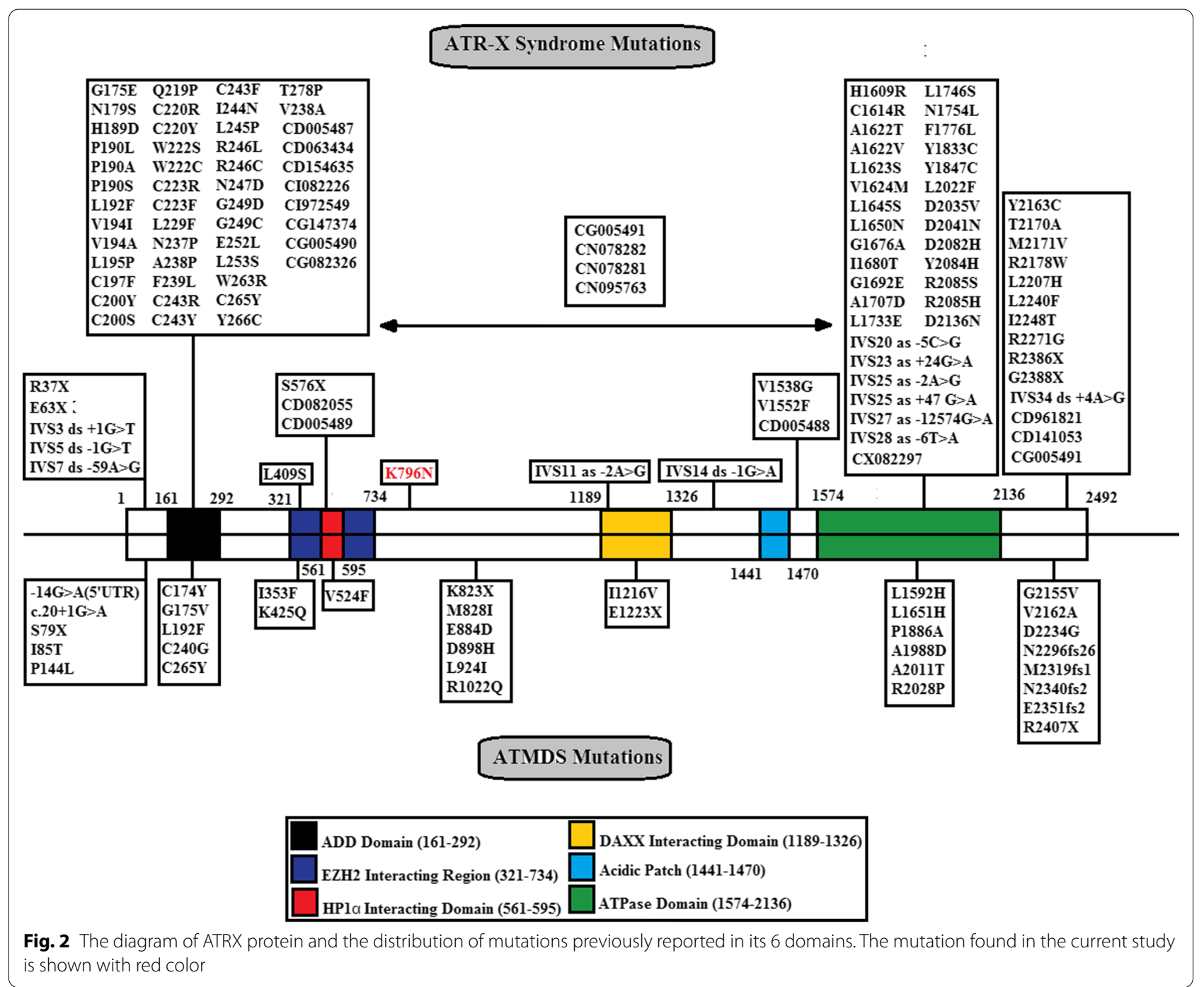

outside of highly conserved domains of ATRX gene. Another possibility is that this mutation is hypomorphic or modifier genetic and epigenetic factors contributing to modulating the effects of this mutation.

\section{Literature review}

\section{Somatic ATRX mutations: ATMDS}

Although aberrant templates of hemoglobin synthesis are almost always inherited, sporadically individuals with previously normal hematology may develop abnormal hemoglobin synthesis as an acquired abnormality [10]. There are a number of reports describing $\alpha$ thalassemia as newly acquired traits in the background of hematologic malignancy [11]. This syndrome was characterized by a marked hypochromic and microcytic anemia with the presence of $\mathrm{HbH}$ ( $\beta 4$ tetramers) and named $\alpha$-thalassemia myelodysplastic syndrome or ATMDS [12]. In the light of these findings, an acquired $\alpha$ thalassemia patients registry was established in the early 1980s [13]. ATMDS predominantly occurs in male (maleto-female ratio greater than 6:1) within the 7th decade of life [12]. Acquired $\alpha$ thalassemia is not limited to the geographical regions in which the inherited forms of $\alpha$ thalassemia are common (e.g., the Mediterranean basin, Southeast Asia, Africa, and Melanesia). Most of ATMDS patients have been of Northern European descent and few Mediterranean and Asian patient have been reported to date. It is feasible that this distribution represents ascertainment bias. If microcytic, hypochromic red cell indices or other signs of thalassemia were apperceived in a patient with hematologic malignancy originating from an area in which inherited forms of thalassemia are common, it is likely that these findings would be related to a previously unrecognized inherited thalassemia, and such a hypothesis would usually be correct. However, rare cases of acquired $\alpha$ thalassemia in patients with these 


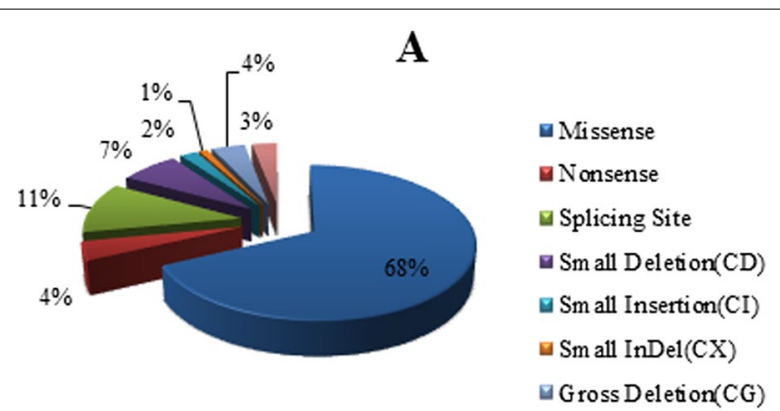

B

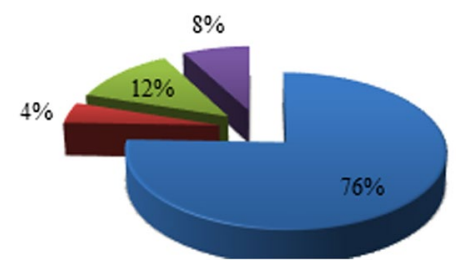

Missense

Nonsense

$\square$ Splicing Site

$\square$ Small Deletion(CD)

Fig. 3 The ATRX gene mutation types reported to date in ATR-X syndrome $(\mathbf{A})$ and ATMDS (B)

areas of the world origin may have been missed. On the other hand, thalassemia red cell indices in an individual with hematologic malignancy who originate from outside of the malaria belt (e.g., from Northern Europe) are unanticipated and should operate further evaluations [12, 13].

At least 2 molecular mechanisms for acquired $\alpha$-thalassemia are presented today: cis-acting defects including acquired deletion of the $\alpha$-globin gene cluster limited to the neoplastic clone and, more commonly, inactivating somatic mutations of the trans-acting regulator of globin gene expression $A T R X$, which cause significant down regulation of $\alpha$-globin gene expression $[11,14]$. It is now demonstrated that most patients with ATMDS have an acquired somatic splicing abnormality or point mutation involving ATRX [11].

\section{ATR-X syndrome}

The rare association of $\alpha$-thalassemia and mental retardation (MR) was presented over 36 years ago in northern European origin patients by Weatherall and colleagues [15]. It is now distinct that this association may occur as a result of two quite distinguished mechanisms; one resulting from large deletions in telomeric region of chromosome 16 ( $\alpha$ thalassemia with retardation on chromosome 16, ATR-16 syndrome OMIM catalog \#141750); the other, caused by mutations in ATRX gene (ATR-X syndrome) [16]. The main clinical features of ATR-X syndrome include severe psychomotor delay, abnormal facial appearance, microcephaly, urogenital abnormalities and a variable degree of $\alpha$ thalassemia, a condition caused by deficient $\alpha$-globin expression $[17,18]$. Patients are characterized during early childhood [17]. ATR-X syndrome is predominant in males, and almost all female carriers have a normal appearance and intellectual ability, although approximately one in four carriers has subtle signs of a-thalassemia, which show an skewed pattern of $\mathrm{X}$-inactivation that lead to the expression of the mutant allele $[17,19]$. The molecular cause of ATR-X is mainly from point mutations in the ATRX gene [12]. More over in some cases the disease appears to raise de novo. In fact, a number of families have been reported in which some or all of the affected members with mutations of $A T R X$, and the characteristic manifestations described previously, have no signs of $\alpha$-thalassemia [17, 20, 21]. In ATR-X patients which have abnormal development of the genitalia (e.g., male pseudohermaphroditism), testis characteristics occurs but the cellular components maturation is failed. It is possible that many of the genes whose regulation is perturbed by $A T R X$ mutations have critical rule in terminal differentiation. It is noteworthy that ATR-X patients do not have an increased incidence of cancer [22].

\section{ATRX gene and protein}

The $A T R X$ gene is located on the Xq13.3; contains 35 exons (Reference sequence NG_008838.3; https://www. ncbi.nlm.nih.gov/). ATRX is a relatively large protein consists of 2492 amino acids $(283 \mathrm{kDa})$. It is a chromatin- associated protein with ATRX-DNMT3-DNMT3L (ADD) domain (encoded by exons 8-10) containing GATA-like zinc finger at the N-terminus, and a long $\mathrm{C}$-terminal that pack together to form a single globular domain containing a helicase/ATP domain (encoded by exons 18-31) [23, 24]. The previous domain formed by seven conserved "helicase" motifs found in DNA-stimulated ATPases and DNA helicases of the SNF2/SWI2 (Switching defective/Sucrose nonfermenting) protein family. The SWI/SNF complexes act as global gene regulators, changing the chromatin structure and altering the accessibility of transcriptin factor to DNA in a subset of specific genes [25]. The ADD and helicase/ATPase are extremely conserved domains. These proteins frequently exist in multicomponent complexes that remodel chromatin and thereby influence multiple epigenetic nuclear processes (e.g., DNA replication, DNA repair, DNA methylation, recombination, transcription). ATRX is widely expressed throughout development [26, 27].

\section{ATRX mutations}

The CpG methylation at heterochromatic loci is disturbed in patients with inherited mutations in $A T R X$ gene. The reality that $A T R X$ mutations affect $\alpha$ but 
apparently not $\beta$ globin expression may be informative. These two gene clusters are embedded in exactly different chromosomal environments, which supports the vision that ATRX influences gene expression via one or more of the epigenetic aspects that distinguish these different regions [28].

Various types of mutations including missense, nonsense, in/del, duplication and splice site have now been reported in ATRX gene in related to ATR-X syndrome (Fi. $2,3)$. It has been hypothesized that in $A T R-X$ syndrome, both copies of gene are inactivated in the patient, one by mutation and the other by X inactivation [29]. Considering the monogenic nature of ATR-X syndrome, it is important to recognize the type of mutations involved and their clinical severity. For example, the ADD domain amino acids mutations lead to more severe psychomotor phenotypes than the helicase domain mutations. Interestingly, a nonsense mutation at residue 37 of ATRX is associated with a milder phenotype than the phenotype created by missense mutations in the ADD and helicase domains [30]. The nonsense mutation at residue 37 is spliced out of a proper subset of transcripts, which partially compensate ATRX protein function. Interestingly, mutations in genes that encode proteins cooperate with ATRX, such as DAXX, have not been identified in patients with ATR-X syndrome [29].

The hematologic phenotype in patients with ATMDS is in general, more severe than that seen in boys with congenital ATR-X syndrome. For example, $\alpha / \beta$ globin synthesis ratios are usually very low $(<0.2$ in $52 \%$ of patients) and the amounts of $\mathrm{HbH}$ are large (median 30\%) in a patients with ATMDS, compared to boys with inherited ATR-X syndrome, who commonly have only mildly reduction in $\alpha / \beta$ synthesis ratios with low and sometimes undetectable amounts of $\mathrm{HbH}$ [31]. The suggestion is that the different in hematologic effects of ATRX mutations in erythroid cells depends on mutation occurrence time during development or, more likely, on the cellular context in which the mutation occurs $[4,31]$.

The patients with the same mutations may have very different degrees of $\alpha$-thalassemia, suggesting that the effect of the ATRX protein on $\alpha$-globin expression may be modified by other genetic and epigenetic factors. This is most clearly illustrated by comparing the hematology phenotype of cases with identical mutations [32].

Here we report a male case with ATRX hereditary mutation manifested mild $\alpha$-thalassemia and no sign of mental retardation, facial dysmorphism, and urogenital abnormalities. His mother was carrier and has a normal appearance and intellect, and no signs of $\alpha$-thalassemia. Our patient has shown neither the severe hematologic signs expected in ATMDS nor the widespread manifestations of the ATR-X syndrome, this may be due to the fact that the mutation in this patient occurs outside of highly conserved domains including ADD and helicase. The other possibility is that this mutation is hypomorphic or modifier genetic and epigenetic factors contributing to modulating the effects of this mutation. Moreover the symptoms of the disease did not occur at birth, as expected in the ATR-X syndrome, and he showed disease symptoms earlier than the age expected foe ATMDS disease.

\section{Conclusions}

According to previous report, inherited ATRX mutations leas to alterations in expression of the $\alpha$-globin genes and are associated with a broad spectrum of clinical presentations, from an asymptomatic $\alpha$-thalassemia trait that is only of reproductive consequences, to the sever $\alpha$-thalassemia mental retardation, facial dysmorphism, and urogenital abnormalities. Therefore a person with a mild $\alpha$-thalassemia phenotype may also have mutation in ATRX gene. As conclusion, it is critical for geneticist and physicians to increase awareness in molecular diagnosis of $\alpha$-thalassemia patients.

\section{Abbreviations \\ AML: Acute myeloid leukemia; ATMDS: Alpha-thalassemia myelodysplastic syndromes; ATRX gene: Alpha-thalassemia/mental retardation syndrome gene; ATR-X syndrome: Alpha-thalassemia/mental retardation syndrome; COSMIC: Catalogue of somatic mutations in cancer; DNMT: DNA methyltrans- ferase; $\mathrm{HbH}$ : Hemoglobin $\mathrm{H}$; Hgb: Hemoglobin; HGMD: Human gene mutation database; MCH: Mean corpuscle hemoglobin; MCV: Mean corpuscular volume; MDS: Myelodysplastic syndromes; MR: Mental retardation; OMIM: Online Mendelian inheritance in man; PCR: Polymerase chain reaction; PLT: Platelets; RBC: Red blood cells; SWI/SNF: SWItch/sucrose non-fermentable; WES: Whole exome sequencing; WBC: White blood cells.}

\section{Acknowledgements}

The authors are thankful to Pasteur Institute of Iran Staff for providing the necessary facilities for the preparation of the paper.

\section{Authors' contributions}

ZS analyzed the data, read past articles, and wrote the manuscript. GR performed laboratory tests and analysis. MH designed the study and edited the manuscript. All authors read and approved the final manuscript.

Funding

There was no funding.

Availability of data and material

Will be available on request.

\section{Declarations}

Ethics approval and consent to participate

This study was reviewed and approved by the Ethics Committee of Pasteur Institute of Iran (Reference number: IR.PII.REC.1397.56). Informed consent for publication was obtained from patient and his family included in the study.

Consent for publication

Not applicable. 


\section{Competing interests}

The authors declare that they have no competing interests.

\section{Author details}

${ }^{1}$ Department of Molecular Medicine, Biotechnology Research Center, Pasteur Institute of Iran, Tehran, Iran. ${ }^{2}$ Pediatric Cell and Gene Therapy Research Center, Tehran University of Medical Science, Tehran, Iran.

Received: 1 July 2021 Accepted: 2 November 2021

Published online: 14 February 2022

\section{References}

1. Brunner AM, Steensma DP (2016) Myelodysplastic syndrome associated with acquired beta thalassemia:"BTMDS." Am J Hematol 91(8):E325

2. Wykretowicz J, Song Y, McKnight B, Choi SW, Magenau J, Takiar R et al (2019) A diagnosis of discernment: Identifying a novel ATRX mutation in myelodysplastic syndrome with acquired a-thalassemia. Cancer Genet 231:36-40

3. Yang Z, Keel SB, Shimamura A, Liu L, Gerds AT, Li HY et al (2016) Delayed globin synthesis leads to excess heme and the macrocytic anemia of Diamond Blackfan anemia and del (5q) myelodysplastic syndrome. Sci Transl Med 8(338):338ra67-ra67

4. Steensma DP, Gibbons RJ, Higgs DR (2005) Acquired a-thalassemia in association with myelodysplastic syndrome and other hematologic malignancies. Blood 105(2):443-452

5. Wang X, Zhao Y, Zhang J, Chen Y (2017) Structural basis for DAXX interaction with ATRX. Protein Cell 8(10):767-771

6. Sáenz SS, Arias B, Hosomichi K, Romero VI (2021) The limits of clinical findings in similar phenotypes, from Carpenter to ATRX syndrome using a whole exome sequencing approach: a case review. Hum Genomics 15(1):1-6

7. Herbaux C, Badens C, Guidez S, Lacoste C, Maboudou P, Rose C (2012) A new ATRX mutation in a patient with acquired a-thalassemia myelodysplastic syndrome. Hemoglobin 36(6):581-585

8. Wilkie A, Gibbons R, Higgs D, Pembrey M (1991) X linked alpha thalassaemia/mental retardation: spectrum of clinical features in three related males. J Med Genet 28(11):738-741

9. Ji J, Quindipan C, Parham D, Shen L, Ruble D, Bootwalla M et al (2017) Inherited germline ATRX mutation in two brothers with ATR-X syndrome and osteosarcoma. Am J Med Genet A 173(5):1390-1395

10. Driscoll MC, Dobkin CS, Alter BP (1989) Gamma delta beta-thalassemia due to a de novo mutation deleting the 5 'beta-globin gene activationregion hypersensitive sites. Proc Natl Acad Sci 86(19):7470-7474

11. Steensma DP, Higgs DR, Fisher CA, Gibbons RJ (2004) Acquired somatic ATRX mutations in myelodysplastic syndrome associated with a thalassemia (ATMDS) convey a more severe hematologic phenotype than germline ATRX mutations. Blood 103(6):2019-2026

12. Farashi S, Harteveld CL (2018) Molecular basis of a-thalassemia. Blood Cells Mol Dis 70:43-53

13. www.imm.ox.ac.uk/groups/mrc_molhaem/home_pages/Higgs/index. html.

14. Clynes D, Higgs DR, Gibbons RJ (2013) The chromatin remodeller ATRX: a repeat offender in human disease. Trends Biochem Sci 38(9):461-466

15. Weatherall D, Higgs D, Bunch C, Old J, Hunt D, Pressley L et al (1981) Hemoglobin $\mathrm{H}$ disease and mental retardation: a new syndrome or a remarkable coincidence? N Engl J Med 305(11):607-612

16. Dyer MA, Qadeer ZA, Valle-Garcia D, Bernstein E (2017) ATRX and DAXX: mechanisms and mutations. Cold Spring Harbor Perspect Med. 7(3):a026567

17 Gibbons RJ (2012) a-Thalassemia, mental retardation, and myelodysplastic syndrome. Cold Spring Harbor Perspect Med 2(10):a011759

18. Shioda N, Yabuki Y, Yamaguchi K, Onozato M, Li Y, Kurosawa K et al (2018) Targeting G-quadruplex DNA as cognitive function therapy for ATR-X syndrome. Nat Med 24(6):802-813

19 Gibbons RJ (2021) ATR-X: ATR-X: a THALASSEMIA/MENTAL RETARDATIONX-LINKED. Cassidy Allanson's Manag Genet Syndromes. https://doi.org/10. 1002/9781119432692.ch7

20. Gibbons RJ, Pellagatti A, Garrick D, Wood WG, Malik N, Ayyub H et al (2003) Identification of acquired somatic mutations in the gene encoding chromatin-remodeling factor ATRX in the a-thalassemia myelodysplasia syndrome (ATMDS). Nat Genet 34(4):446-449

21. Villard L, Gecz J, Mattéi JF, Fontés M, Saugier-Veber P, Munnich A et al (1996) XNP mutation in a large family with Juberg-Marsidi syndrome. Nat Genet 12(4):359-360

22. De La Fuente R, Baumann C, Viveiros MM (2011) Role of ATRX in chromatin structure and function: implications for chromosome instability and human disease. Reproduction (Cambr, Engl) 142(2):221

23. Barresi V, Ragusa A, Fichera M, Musso N, Castiglia L, Rappazzo G et al (2010) Decreased expression of GRAF1/OPHN-1-L in the X-linked alpha thalassemia mental retardation syndrome. BMC Med Genomics 3(1):1-11

24. https://www.ncbi.nlm.nih.gov/.

25. Ratnakumar K, Bernstein E (2013) ATRX: the case of a peculiar chromatin remodeler. Epigenetics 8(1):3-9

26 Wong LH, McGhie JD, Sim M, Anderson MA, Ahn S, Hannan RD et al (2010) ATRX interacts with H3. 3 in maintaining telomere structural integrity in pluripotent embryonic stem cells. Genome Res 20(3):351-360

27. Valenzuela M, Amato R, Sgura A, Antoccia A, Berardinelli F (2021) The multiple facets of ATRX protein. Cancers 13(9):2211

28. Chaudhary S, Dhawan D, Sojitra N, Chauhan P, Chandratre K, Chaudhary PS et al (2017) Whole gene sequencing based screening approach to detect $\beta$-thalassemia mutations. Biol Med 9:2

29. He J, Mansouri A, Das S (2018) Alpha thalassemia/mental retardation syndrome X-linked, the alternative lengthening of telomere phenotype, and gliomagenesis: current understandings and future potential. Front Oncol 7:322

30. Dyer M, Qadeer Z, Valle-Garcia D, Bernstein E (2017) ATRX and DAXX: mechanisms and mutations. Cold Spring Harb Perspect Med 7:a026567

31. Higgs DR (2004) Ham-Wasserman lecture: gene regulation in hematopoiesis: new lessons from thalassemia. ASH Educ Progr Book 2004(1):1-13

32. Law MJ, Lower KM, Voon HP, Hughes JR, Garrick D, Viprakasit V et al (2010) ATR-X syndrome protein targets tandem repeats and influences allelespecific expression in a size-dependent manner. Cell 143(3):367-378

\section{Publisher's Note}

Springer Nature remains neutral with regard to jurisdictional claims in published maps and institutional affiliations.

\section{Submit your manuscript to a SpringerOpen ${ }^{\circ}$ journal and benefit from:}

- Convenient online submission

- Rigorous peer review

- Open access: articles freely available online

- High visibility within the field

- Retaining the copyright to your article

Submit your next manuscript at springeropen.com 